\title{
Research on Form Innovation Design and Implementation of Secondary Reconstruction for Cool Core Fabric
}

\author{
Jun Liang ${ }^{1}$, \\ ${ }^{1}$ School of Art,Northeast Dianli \\ University, Jilin,China
}

\author{
Yueming Yang ${ }^{1}$ \\ ${ }^{1}$ School of Art,Northeast Dianli \\ University,Jilin,China
}

\begin{abstract}
In this paper, we conduct theoretical analysis and literature review applications on the form innovation design and implementation of secondary reconstruction for cool core fabric. At present, due to the development of The Times and the progress of science and technology, people's aesthetic concept also transformed, functional and decorative fabric art aesthetic has been raised to an important level, but also strong. Second fabric design is refers to the use of various traditional and high-tech means to the existing fabric to open out the design of the processing, make its surface rich visual texture and touch skin texture. Our method is effective and novel, we will try to make it into real-world applications in the future.
\end{abstract}

Keywords: Cool Core Fabric; Secondary Design and Implementation; Reconstruction Procedure.

\section{Introduction}

Decoration material is the material base of decorative fabric design which is accomplished. As a result, the performance of the decoration materials must have full understanding, to master the performance of it. According to the actual situation of decorative objects and specific environmental conditions appropriately, use adornment material to make all the fully play the role of the adornment material, to maximize the each adornment material to make, so that the decoration materials design can fully show the content. In the use of the fabric, for the design and application of the same fabric, there are various ways of its taken and it reflects the contemporary designer in art expression form of the rich and profound artistic language. Decorative fabrics can be divided into the following kinds: cotton fabrics, silk textile fabrics, woolen fabrics, chemical fiber textile fabrics and non-porous chemical fiber fabrics and so on. Cotton fabric has better moisture absorption and permeability, but its shrinkage, alterative and relatively poor corrosion resistance; Silk textile fabrics, soft light, color, delicate and charming, has the advantages of absorbing water permeability, but easy to crinkle fade; Woolen fabric has good heat preservation performance, wear resistance and resilience is stronger, but its thermal performance is poorer, easy shrink and plush ball; With the development of science and technology, chemical fiber textile fabrics and non-porous emerge in endlessly and chemical fiber fabrics were listed, they not only have the fine qualities of traditional textile fabrics, also has the advantages of bright colors, strong corrosion resistance, but poor generally poor permeability. At present, due to the development of The Times and the progress of science and technology, people's aesthetic concept also transformed, functional and decorative fabric art aesthetic has been raised to an important level, but also strong. Design the healthy and comfortable convenient and fashionable fabrics are the inevitable direction of decorative fabric design. Main characteristics of decorative fabric has changed, the fabric design requirements for comfort, for the sake of customers to meet the 
demand of the times, decorative fabric design also requires that achieve high grade. Therefore, the function of the fabric is in accordance with the time feature and style is the combination of high-grade decorative fabric design era characteristics [1-2].

In this paper, we conduct theoretical analysis on the form innovation design and implementation of secondary reconstruction for cool core fabric. Second fabric design is refers to the use of various traditional and high-tech means to the existing fabric to open out the design of the processing, make its surface rich visual texture and touch skin texture. Recreation of art gradually as a new breakthrough, costume design and become an important means to improve clothing products value-added. Secondary design, fabric can also be referred to as the fabrics of reengineering, refers to the designer according to the needs of costume design, the ready-made fabric processing and transformation, to produce the delicate and elegant artistic charm. After the second design of fabric more to comply with the idea of the designer in the heart, because it itself already finished half of costume design work, also can bring more inspiration for fashion designers and creative passion. Coordination between fabrics and clothing design is vital part of the costume design, fabric is not only the material basis for the clothing modeling, is also the important form of plastic arts, such as concise design modeling can be combined with depth and texture of fabric, to show its strong visual impact, and pure, exquisite material can use exaggeration and changeful modeling, improper together, the performance of the visual effects in terms of primary and secondary and personality, and cannot be achieved in the form and the unity on the style. In the figure one, we show the sample cool core fabric based clothing [3-4].

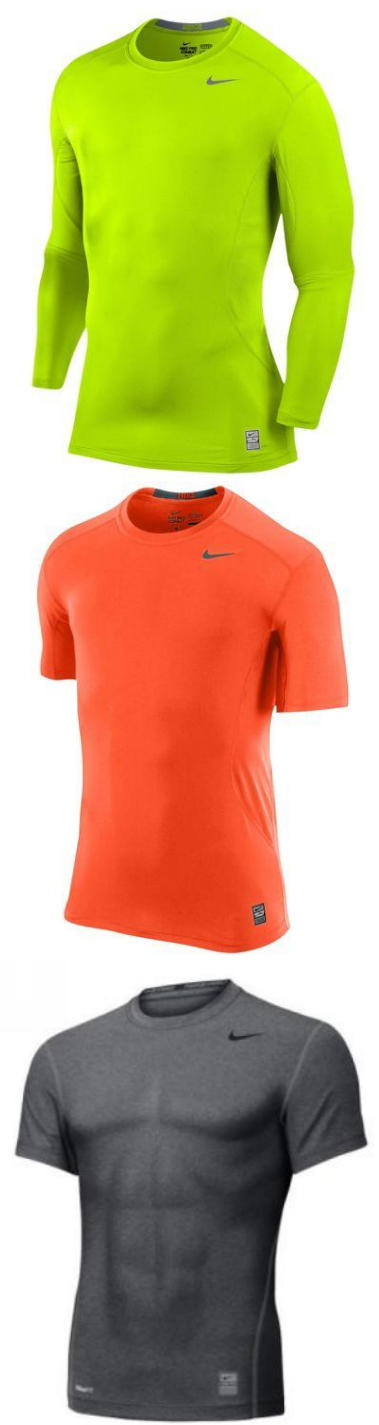

Fig 1. The Sample Cool Core Fabric based Clothing

\section{The Principles of the Our Methodology}

The Concepts of Secondary Reconstruction. The combination of fabric and then create gradually become fashion design and a new breakthrough and enhancing the added value of the clothing products is an important means of the combination of fabric is the fabric itself between the fabrics and fabrics and the combination between a variety of fabrics to reflect the diversity of fabrics to express strengthen the innovation of clothing on the vision express the aesthetic effect of rich and complex re-invention of fabric are the fabric by 
using traditional and high-tech means of shape again shows its rich connotation in the costume design. Use their existing fabric by means of a variety of clothing technology, open design process to make its surface rich visual texture and tactile texture is called the secondary design on the clothing visual texture of fabrics of mainly by all kinds of apparel fabrics have different color design and patterns to reflect the tactile texture is actual touch by us and give us different psychological feeling of skin texture, such as rough with the smooth and soft and hard exquisite and light texture and visual texture and thick touch the biggest distinction is the change of depth in space and form. The methodology could be separated into the following. (1) Dyeing. Here refers to dyeing, garment or garment piece dyeing of distinctive rich color effect like gradient local dyeing dyed by hand is given priority to with heavy manual lasting appeal of the special methods. Manual way of painting can be design also can be random shape usually have a tool drawing method finger ejection method splash-ink ornament spray method draw the outline of dark embossed method combined with the hand-painted. (2) Printing. Printed on clothes usually adopt the following two ways in general is given priority to with local printing screen printing using mesh plate making printing paste outflow from the mesh into the fabric pattern is formed on transfer printing method by hot-pressing will be transferred to the fabric that the pattern of paper there are ready-made on transfer printing paper can also be designed through computer pattern through the transfer printing machine photographically reproduced effect. (3) Multiple-layered design. Through the smoke plait to pinch pleated folds contraction joint quilting embossed craft gimmick change fabrics such as woven morphology formation on the surface of the original embossing and stereo effect and has a strong sense of touch. (4) Adding design. Nail seam on the fabric by post hang embroidery flocking glue hot-pressing method to add the same or different material with special aesthetic feeling of fabrics of different material can form different contrast effect. (5) Subtraction design. Damage the surface of the existing fabric has the surface characteristics, irregular or incomplete methods have hollow shear for cutting the reel off raw silk from cocoons burn flower burnt-out tear remaining hair, make the material presents sand washing process in the use of the denim canvas has for a long time in addition to the integral style finishing on fabric in the clothing of local sand can wash out all sorts of the shape of the light color design. (6) Reverse thinking design. In order to show the essence of fabrics and clothing fabrics like unfinished goods in treating sex is better than the design itself to do sew a seam on the front of clothing and usually not a prize edge processing to reserve the unfinished is thicker. Next section will show the proposed novel approach.

The Proposed Novel Design Pattern. Color vision has a direct impact on the human body, plays a very important role in the clothing. Although fabric color varieties on the market, but still can't meet the personalized requirements of people. On the color transformation, often use tie-dye, batik, hand-painted, splicing technique, digital painting, etc. To dye and batik are in harmony with the fabric dye boiled in hot water, make the fabric to color, use of taped parts, waxing fabric shading fabric characteristics, makes the design and color of the fabric showing a personality is distinct, hand-painted and prints are redesigning design, joining together are the same fabric, different fabrics, different design, colors, stitching restructuring in together, and often and other technique combined with the application. In changing patterns of the fabric is based on the existing fabric to modify the original design, to create new patterns. In changing patterns of should mainly consider the combination of point, line and plane design and color collocation relations, avoid new patterns and disorderly. Nowadays the society is becoming more and more complex and internationalization, the global hot topic is dependent on the energy conservation and 
environmental protection. Stylist is walking in the front of the pursuit of its low carbon environmental functional fabrics. In the modern material design teaching, it also becomes one of the more and more important topics. Changing the clothing science and technology, it has scalability in fabrics, permeability, thermal regulatory and radiation, antimicrobial properties, etc, has brought many benefits. Comprehensive reform refers to the integrated application of color, texture, grain appearance makeover. Fabric processing form is varied, in order to achieve good results and often from various aspects of the comprehensive reform them. In practice, designers tend to different processing all kinds of fabrics, and then combined by using the method of joining together. This will achieve color, texture, pattern and so on various aspects collocation, so as to be rich and strong texture contrast.

Fabric are many factors that affect secondary transformation, transformation method are many, is often a combination of these factors and method application, comprehensive treatment of fabric. But in the practice of various methods cannot optional collocation, we should scientifically according to the characteristic of fabrics, clothing style and follow certain principles. Each fabric has its own characteristics, in color and thickness degree and intensity of each are not identical. Functional textile development has become the worldwide hot spots, expand the functionality of products become the important way to improve product added value. Jeans back into the market, for example, after a soft, anti-fouling, late again, make its coruscate gives a distinctive appearance and the performance of the new, become the new age women are following the popular material of high fashion; The rapid development of elastic fabric is due to elastic fiber is research and development, to join the composition such as the sample in many high-grade fabrics, make it not only has good lubricious feeling, also become more soft, is one of the top fashion now custom common fabrics. We are in changing on fabric, should make clear the fabric characteristics, according to these characteristics, and then with the scientific process, to achieve the desired effect function is one of the basic requirements of clothing, costume design, to consider its functionality. The fabric is designed for the implementation effect in changing and service, guarantee the functional is inevitable. The principles of the design are shown in the following section.

The Principles of the Design. Fabric as one of the three elements of clothing, not only can interpret clothing style and features, and directly influences the effect of color and modeling the performance of the clothing. Fabric is closely related to fashion design, costume design to obtain a good effect, must give full play to the performance and characteristics of fabric, fabric characteristics and the perfect combination of clothing and overall modeling style bring out the best in each other. Reconstruction after the fabric has the change, vivid, three-dimensional, diverse and complex. If use reengineering fabric for garment design, it can be used for local clothing, namely clothing design point, and design style photograph echo, to make the finishing point of aesthetic feeling; It also can be used in the design of the clothing modeling, the fabrics of clothing emphasizes the reengineering of novel and unique charm. A result of the fabric after reengineering already have very strong adornment sex and artistic quality, even to the simplest design also can appear delicate and vivid. But local application must be linked and the overall design, reconstruction after the fabrics in color, shape, etc, must be in harmony and the whole piece of clothing style and model. In the following figure two, we show and illustrate the sample reconstruction element for designing. 


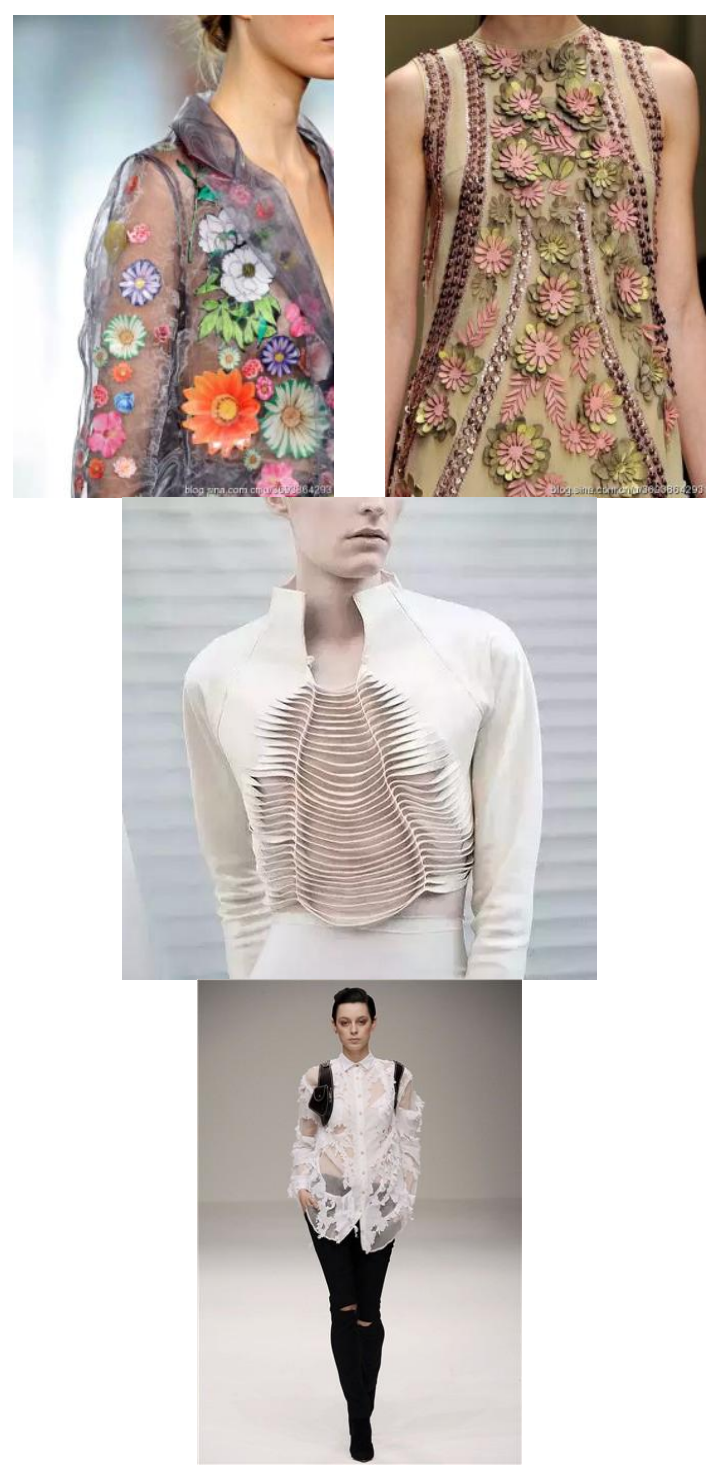

Fig 2. The Sample Reconstruction Element for Designing the Cool Core Fabric

\section{CONCLUSIONS}

In this paper, we conduct theoretical analysis and literature review applications on the form innovation design and implementation of secondary reconstruction for cool core fabric.
The combination of fabric and then create gradually become fashion design and a new breakthrough and enhancing the added value of the clothing products is an important means of the combination of fabric. We are in changing on fabric, should make clear the fabric characteristics, according to these characteristics, and then with the scientific process, to achieve the desired effect function is one of the basic requirements of clothing, costume design, to consider its functionality. The designed pattern is novel and effective, in the future, we plan to do more literature review to modify the current designing approach.

\section{References}

[1] S, O., Y, K., N, S., T, O., \& N., M. (2014). Secondary head and neck reconstruction using free flap to improve the postoperative function or appearance of cancer survivors.. Microsurgery, 34, 2, 122-128.

[2] Sadigh, P. L., Hsieh, C., Feng, G., Shih, H., \& Jeng, S. (2015). A "green" approach to secondary reconstruction: the concept of the recycle flap and a classification.. Plastic \& Reconstructive.

[3] Allan, B. J., Haren, R. M. V., Wang, B., \& Thaller, S. (2015). Secondary soft tissue reconstruction. Ferraros Fundamentals of Maxillofacial Surgery.

[4] Lee, H. J., Choi, J. W., \& Chung, I. W. (2014). Secondary skull reconstruction with autogenous split calvarial bone grafts versus nonautogenous materials.. Journal of Craniofacial Surgery, 25, 4, 1337-1340. 